\title{
RUPTURE PROCESSES IN POLYMERS
}

\author{
THOR L. SMITH \\ I.B.M. Research Laboratory, San Jose, California 95114, U.S.A.
}

\begin{abstract}
The strength and toughness of polymers depend on stress-induced dissipative and disruptive processes exemplified by viscoelastic and plastic deformations, cavitation, and crack growth. High strength necessitates molecular immobility; toughness results from mobility and the dissipation of stored elastic energy. These concepts are discussed in terms of the dissipative and disruptive processes that occur under stress in various types of elastomeric and plastic materials. The role of structure at the molecular and supermolecular levels is considered along with that of elastic constraints which, by altering the stress state, modify strength and toughness. To have high toughness and strength, a material must contain a dispersed phase. When effective, the dispersed phase acts to increase the dissipation of energy, often through controlled structural break-down, and to impede the development of large cracks.
\end{abstract}

\section{INTRODUCTION}

Macroscopic rupture is commonly, if not always, the culmination of various stress-induced dissipative and disruptive processes that occur in localized regions throughout a specimen. The initiation and subsequent development of such processes are difficult to investigate because they occur on a microscopic scale and involve only a small fraction of the material in a test specimen. Consequently, the effect of microscopic breakdown on mechanical response characteristics is often obscured by deformational processes that occur rather uniformly throughout a specimen. Hence, to a casual observer, rupture processes ordinarily proceed unnoticed until catastrophic crack propagation or some other instability develops.

In this paper a discussion is given of strength, toughness, and extensibility of polymers. Strength refers to the stress sustained by a specimen immediately prior to macroscopic rupture or, in some instances, to a yielding process. Toughness is the energy required to rupture a specimen; it may also be defined as the resistance to further growth of a crack. Extensibility refers to the elongation, or strain, at the instant of rupture. To consider these properties, attention is focussed on: (1) the various disruptive processes that can occur in a stressed specimen and their dependence on structural characteristics and test conditions; and (2) the conditions that lead to the terminal phase in the rupture process, namely, the self-sustained high-speed propagation of a crack. Although definitive information on these topics is somewhat limited, various concepts and theories have been advanced that provide a 
qualitative, or semi-quantitative, explanation of the principal aspects of rupture.

\section{GENERALIZATIONS}

High strength necessitates molecular immobility. If polymeric segments are highly mobile, a high stress cannot be sustained by a specimen owing to stress-relief mechanisms. In contrast, mobility is a necessary but not a sufficient condition for high extensibility; other requirements are energy dissipative mechanisms along with a low density of crosslinks and permanently entangled chains. Because toughness depends, in effect, on both stress and extensibility, high toughness necessitates an optimized mobility and localized processes that relieve stress concentrations and thus prevent the development of a catastrophic disruptive process. Because localized flow dissipates stored elastic energy, it follows that the source of toughness is energy dissipation.

These generalizations are supported by the well-known fact that the strongest materials, e.g. graphite whiskers, drawn high-carbon steel, and glass fibres, are extremely brittle. The high strength of such materials is a reflection of the vanishingly small mobility of their structural elements. Upon modifying structure to impart mobility, the toughness of the resulting material may be increased but its strength is reduced. Although this inverse relation between strength and toughness is commonly observed, it is far from being universally true. For example, structural changes that yield a substantial increase in the strength of an elastomer generally give an increase in tear strength, a measure of toughness.

When a polymer is deformed, various time-dependent processes begin that tend to reduce the stored elastic energy. These processes may be either destructive or non-destructive, depending on whether or not primary chemical bonds are broken. A viscoelastic deformation involves structural rearrangements that are normally non-destructive. Examples are: the stressbiased diffusion of polymer chains or segments; the rearrangement of side groups or short segments; and, for certain two-phase materials, the deformation and rotation of crystalline or glassy domains. Under many, but not all, test conditions, these structural rearrangements occur homogeneously; that is, the changes in any small volume element are the same as those in any other volume element. In contrast, rupture is a dilatational process that involves cavitation and crack growth; chemical bonds are broken and morphological units are disrupted. Such processes are nonhomogeneous; that is, they develop somewhat randomly in localized regions throughout a specimen.

\section{DIRECT EVIDENCE FOR DESTRUCTIVE PROCESSES}

It is quite apparent that rupture entails the breakage of primary chemical bonds. Until recently, however, there was no direct experimental evidence that bonds in a stressed specimen are in fact broken and that such a disruptive process commonly begins long before macroscopic rupture occurs. In a recent study ${ }^{1}$, specimens of poly (methyl methacrylate), polystyrene, 
and polypropylene were stressed in a mass spectrometer. As soon as the stress was applied, degradation products were detected, and their evolution rate was observed to increase precipitously as the time for macroscopic failure was approached. Furthermore, the evolved products were found to be substantially identical to those produced during the thermal degradation of unstressed specimens.

In another study ${ }^{2}$, specimens of an unfilled vulcanizate of natural rubber were stretched 100 per cent at room temperature and then cooled to $-120^{\circ}$; the resulting oriented material was noncrystalline. When stretched at $-120^{\circ}$, the specimens underwent yielding and subsequently ruptured at about 200 per cent elongation, in excess of the initial 100 per cent. After the yield point was reached, substantial quantities of hydrogen were evolved, presumably because polymeric chains were being broken. The resulting radicals recombined giving an increased crosslink density. Data from equilibrium swelling tests showed that the molecular weight between crosslinks was reduced from 6650 to 4900 by the drawing process.

The formation of free radicals in stressed fibres has been investigated by electron spin resonance (ESR) spectroscopy ${ }^{3}$. In fibres of nylon 6 and natural silk thread, radicals were detected at stresses considerably below the breaking stress. The concentration of radicals increased with the applied stress, or with the time under a constant stress, especially as the rupture point was approached. Furthermore, the rate of radical production in nylon 6 specimens under constant stress was correlated quantitatively with specimen lifetime, measured under different loads at temperatures from $-50^{\circ}$ to $50^{\circ}$.

In a similar study ${ }^{4}$, rods of nylon 6 and 66 were cold drawn under vacuum at room temperature in the cavity of an ESR spectrometer. Although a specimen was transformed into a highly anisotropic structure, no radicals were detected during the plastic deformation. However, when fibres of nylon 66 were stretched and maintained at constant elongation, not only were radicals formed but also their concentration increased continuously during a 200-minute test period, another verification of the kinetic nature of the stress-induced degradation process. In a specimen stretched essentially to rupture, the concentration of radicals was estimated to be $10^{17} \mathrm{~cm}^{-3}$. From this observation, it was deduced that the number of bonds broken is 100 -fold greater than the number of chains passing through a plane perpendicular to the stretch direction. It was hence concluded that chain rupture is not confined to the rupture plane but occurs throughout the entire volume of a stressed specimen.

The above-mentioned investigations, along with additional studies ${ }^{5,6}$ of stress-induced radical formation, provide direct evidence that chemical bonds are often broken in a stressed specimen and that the rate of bond rupture increases catastrophically as the time for macroscopic rupture is approached.

\section{STEPS IN THE RUPTURE PROCESS}

Although resulting from destructive processes, bond rupture per se has little or no bearing on strength characteristics; other dissipative and disruptive processes have a controlling effect. Rupture may be envisaged to 
involve three steps or phases. The first, or initiation, phase is the formation of a cavity or crack that begins to increase in size. The second step is the slow growth of a crack until an instability develops. And the third, or terminal, phase is the self-sustained high-speed propagation of a crack. Although many microcracks commonly develop within a stressed specimen, it is customary to focus attention on the particular crack that eventually becomes unstable and propagates across the specimen. In actuality, however, the first and second phase of the rupture process undoubtedly occur simultaneously. The growth rate of a crack may be affected by the formation and enlargement of other cracks. Specifically, the formation of new cavities and cracks can retard, or arrest completely, the growth of existing cracks ${ }^{7,8}$. Eventually, however, one or more cracks become unstable and high-speed growth ensues.

In the first phase of the rupture process, cracks may develop from stressinduced, or possibly pre-existent, cavities. All materials contain, or develop under stress, heterogeneities that give regions of stress concentration. These regions are potential sites for the formation of cavities, which under certain conditions are transformed into cracks. In highly brittle materials, cracks commonly grow from surface flaws or nicks; the strength of glass fibres, for example, is determined in large measure by surface defects ${ }^{9}$. In softer materials, cavitation leading to crack growth can occur in regions of triaxial tension within the specimen. For example, it has been observed ${ }^{10}$ that cracks initiate within specimens of unfilled and filled elastomers about as frequently as on the surface.

Certain aspects of crack growth can be illustrated by considering a single crack in a large sheet of an ideally elastic (brittle) material and also in an elastic-plastic material. Specifically, consider that the major axis of the crack (the $x$-axis) is parallel to the edges of the sheet at which a normal stress
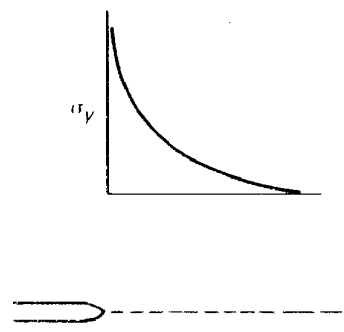

(a) Crack growth in an ideal elastic (brittle) material
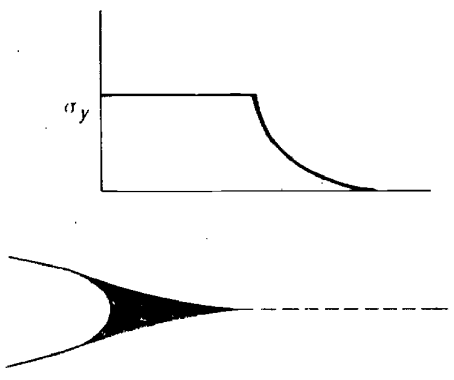

(b) Crack growth in an ideal elastic-plastic material

Figure 1. Schematic diagrams to illustrate the stress in front of a crack in an ideal elastic material and an elastic-plastic material.

is applied. For a crack having a tip of finite radius (elliptical crack), a calculation has been made ${ }^{11}$ of the stress components $\sigma_{y}$ and $\sigma_{x}$ (parallel and perpendicular, respectively, to the applied stress) in the vicinity of the tip. 
Along the $x$-axis $\sigma_{y}$ is a maximum at the crack tip and decreases until it eventually attains the value in the bulk of the specimen, as indicated schematically in Figure 1a. Again, with increasing distance along the $x$-axis, $\sigma_{x}$ increases from zero at the crack tip, attains a maximum, and then decreases to zero. The maximum value of $\sigma_{x}$, which is about one-fifth of the maximum value of $\sigma_{y}$, occurs at a distance in front of the crack that roughly equals the crack tip radius. Hence, triaxial tension exists on and in the vicinity of, the $x$-axis away from the crack tip.

When the stress near the crack tip (Figure 1a) reaches the cohesive strength of the material, the crack will begin to grow, quickly reaching a limiting velocity which is approximately one-half of the velocity of a shear wave. In this idealized process, stored elastic energy is converted into kinetic and surface energy, the latter being the energy required to rupture the bonds along the fracture plane. In terms of the well-known Griffith criterion, the crack will become unstable and propagate when the decrease in stored elastic energy resulting from an increase in crack dimensions becomes equal to the surface energy. The Griffith criterion can be written in the form:

$$
W C=K \mathscr{G}
$$

where $W$ is the density of elastic energy some distance from the crack, $C$ is the half-length of the crack, and $K$ is a dimensionless geometrical factor. In the Griffith equation, $\mathscr{G}$ is the surface energy and $K=1 / \pi$.

Now consider a crack in a sheet of an ideal elastic-plastic material that is subjected to a progressively increasing stress. When $\sigma_{y}$ at the crack tip becomes equal to the yield stress, a plastic deformation begins, leading to a rounded crack tip and a wedge of plastically deformed material ahead of the crack, indicated in Figure $1 b$. Eventually--possibly when a plastic strain criterion is satisfied--the crack begins to grow. As the crack grows, a sizeable volume of material on both sides of the fracture plane is plastically deformed, dissipating considerable elastic energy.

The criterion for high-speed growth is again given by equation 1, except that $\mathscr{G}$ can now be called the fracture surface energy, which is the work required to produce a unit area of new surface. In this idealized process, the energy required to rupture valence bonds along the fracture plane is negligibly small compared to the work of plastic deformation.

It is generally found that a crack grows slowly, but at a progressively increasing rate, until a constant, high velocity results ${ }^{12}$. In elastomers, the slow-growth phase may proceed for a prolonged period and the transition to high-speed growth occurs rather suddenly ${ }^{8,13-15}$. The fracture surface formed during slow growth is somewhat rough ${ }^{13,14}$, reflecting the development of a fibrous structure near the crack tip, probably resulting from cavitation in the region of triaxial tension ahead of the crack tip. At high speeds, the fracture surface formed is relatively smooth owing to a sharpening of the crack tip. For hard plastics ${ }^{16}$, the smooth portion (mirror area) of the fracture surface occurs in the vicinity of the fracture initiation site; surface roughness results from the propagation of secondary cracks and from the bifurcation of cracks arising from a high release rate of strain energy. 
The transition from low- to high-speed growth is commonly considered $^{14,15}$ to occur when

$$
(W C)_{c} \geqslant K \mathscr{G}_{c}
$$

Although this equation is similar to the Griffith criterion, the symbols $K$ and $\mathscr{G}_{c}$ are interpreted differently. The dimensionless factor $K$ depends somewhat on the magnitude of the deformation near the crack. The quantity $\mathscr{G}$ is again the energy required to create a unit area of new surface; it depends on the nature of the dissipative processes that accompany crack growth. Hence, $\mathscr{G}$ depends not only on the properties of the material but also on the stress state and the rate of crack growth. In a subsequent section, factors that affect $\mathscr{G}$, especially for elastomeric materials, will be considered in more detail.

\section{DILATATION AND CAVITATION}

The essence of rupture is dilatation resulting from cavitation and crack growth. Hence, it is instructive to consider homogeneous dilatation under several types of tensile deformations and also the types of instabilities that can result.

Several pure homogeneous tensile deformations are defined in Table 1 in terms of the principal stresses and strains. Simple tension and equal (uniform)

Table 1. Definition of several types of tensile deformations

\begin{tabular}{l|c|c|c|c|c|c}
\hline Deformation & $\varepsilon_{1}$ & $\varepsilon_{2}$ & $\varepsilon_{3}$ & $\sigma_{1}$ & $\sigma_{2}$ & $\sigma_{3}$ \\
\hline ST & $\varepsilon$ & $-v \varepsilon$ & $-v \varepsilon$ & $\sigma$ & 0 & 0 \\
CBT & $\varepsilon$ & 0 & $-v \varepsilon /(1-v)$ & $\sigma$ & $v \sigma$ & 0 \\
EBT & $\varepsilon$ & $\varepsilon$ & $-2 v \varepsilon /(1-v)$ & $\sigma$ & $\sigma$ & 0 \\
CTT & $\varepsilon$ & 0 & 0 & $\sigma$ & $v \sigma /(1-v)$ & $v \sigma /(1-v)$ \\
& & & & & & \\
\hline
\end{tabular}

biaxial tension are designated by ST and EBT, respectively. When a specimen is subjected to uniaxial tension while the lateral contraction in one direction is constrained, a state of biaxial tension is produced, which is here termed constrained biaxial tension (CBT). Similarly, if the lateral contraction

Table 2. Dilatation in tension

\begin{tabular}{l|l|l|l|c}
\hline \multirow{2}{*}{ Deformation } & $\mathrm{e}=\frac{\Delta V}{V_{0}}$ & \multicolumn{3}{|c}{$e / e_{\mathrm{sT}}$} \\
\cline { 3 - 5 } & & $v=v$ & $v=0.25$ & $v \cong 0.5$ \\
\hline $\mathrm{ST}$ & $\varepsilon(1-2 v)$ & 1 & 1 & 1 \\
$\mathrm{CBT}$ & $\varepsilon(1-2 v) /(1-v)$ & $1 /(1-v)$ & $1 \cdot 33$ & 2 \\
EBT & $2 \varepsilon(1-2 v) /(1-v)$ & $2 /(1-v)$ & $2 \cdot 67$ & 4 \\
CTT & $\varepsilon$ & $1 /(1-2 v)$ & 2 & $\infty$ \\
& & & & \\
\hline
\end{tabular}


in both directions perpendicular to the applied load is constrained, a state of triaxial tension, here termed constrained triaxial tension (CTT), is produced. The formulae in Tables 1 and 2 are based on classical elasticity, which is applicable precisely only in the limit of zero strain. Hence, the relations that contain Poisson's ratio are not quantiatively correct at large deformations, although they are adequate to illustrate certain trends.

The second column of Table 2 gives the dilation, $e=\Delta V / V o$, effected by a tensile strain $\varepsilon$. The last columns give $e / e_{S T}$ for two values of Poisson's ratio, where $e_{S T}$ is the dilation in simple tension. As expected, the CBT, EBT, and CTT deformations are considerably more dilatative than ST, especially for elastomers for which $v \cong 0 \cdot 5$. For an elastomer, the CTT deformation is equal triaxial (hydrostatic) tension, as can be seen by substituting $v=0.5$ in the relations in Table 1.

Polymers, as well as other materials, are quite resistive to dilatation, an indication being their bulk moduli which are in the range $10^{10}$ to $10^{11}$ dynes $/ \mathrm{cm}^{2}$. It is thus not surprising that an instability develops when the imposed homogeneous dilatation becomes unduly large. The instability may be manifest by: (1) blushing, which is the formation of numerous small cavities throughout a specimen; (2) crazing, which is the formation of an oriented porous structure in limited regions of the specimen; and (3) rupture. The specimen ruptures if the cavities or crazes that tend to form are unstable. Each of these instabilities may be initiated by stress concentrations near the flaws or heterogeneities that are present in all materials.

Various experimental observations on microfailures (instabilities) within stressed specimens have been reviewed by Rosen ${ }^{17}$. In addition to microscopic examination ${ }^{7}$, information on microfailures has been obtained from studies of the stress and time dependence of the intensity of reflected light ${ }^{18}$, the enhanced permeability of helium and nitrogen gases through specimens deformed in tension ${ }^{19,20}$, and light and small-angle $x$-ray scattering ${ }^{21}$.

The formation of numerous cavities--a process called blushing owing to the turbid appearance of the specimen--apparently necessitates an optimum mobility of polymeric chains. For example, polystyrene and poly(methyl methacrylate) blush only when deformed under suitable conditions at $60-80^{\circ}$. From a study ${ }^{21}$ of blushed specimens by light and $\mathrm{x}$-ray scattering (small angle), it was concluded that about $10^{12}$ cavities $/ \mathrm{cm}^{3}$ exist and that their radii are in the range 400 to $1200 \AA$, depending on the material.

The occurrence of permanent damage, and thus bond rupture, during blushing is indicated by a study ${ }^{22}$ of creep and creep-rupture of cellulose acetate under conditions that produce blushing. When a specimen from an interrupted creep test was heated at $70^{\circ}$, its original dimensions and appearance were regained. However, when the specimen was tested again, the creep rate was found to be greater than originally and the time-to-break to be less than that for a fresh specimen. In fact, the time-to-break for a fresh specimen under an uninterrupted load was sensibly the same as the sum of the time under load before and after the other specimen was annealed.

The rupture of elastomers under triaxial tension has been studied ${ }^{23,24}$. Information was not obtained on the origin of small cavities but only on the conditions and manner in which cracks propagates radially from an enlarged cavity ${ }^{24}$ (about 25 microns in diameter). To obtain triaxial 
tension, a thin disc of rubber was bonded between metal ${ }^{23}$, or Lucite ${ }^{24}$, platens and subjected to uniaxial tension. Because the specimen could not contract perpendicular to the applied load, triaxial tension resulted; at small applied strains, macroscopic failure occurred in the centre of the specimen. The nominal stress (applied load divided by the cross-sectional area of the specimen) was found to increase linearly with the Young's modulus of the specimen, as shown in Figure 2. Presumably, very small cavities first form in the specimen. Under the imposed triaxial tension, a cavity enlarges until the extension on the surface of the cavity becomes very large, causing the surface

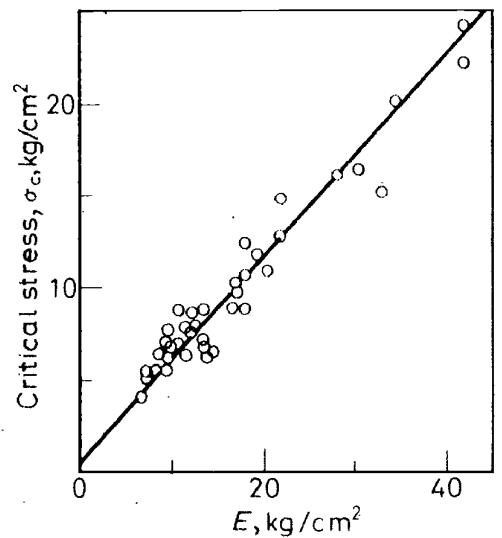

Figure 2. Relation of Young's modulus $E$ for elastomers to the uniaxial tensile stress at the instant of internal rupture under triaxial tension (from Gent and Lindley ${ }^{23}$ ).

to rupture and cracks to propagate radially. Taking this picture as a model, Gent and Lindley ${ }^{23}$ analyzed mathematically the enlargement of a cavity under hydrostatic tension. They found that the hydrostatic tension at which the extension ratio becomes very large, and thus at which the cavity ruptures, increases in direct proportion to Young's modulus, in agreement with experimental observations. Subsequently, the instability of a spherical

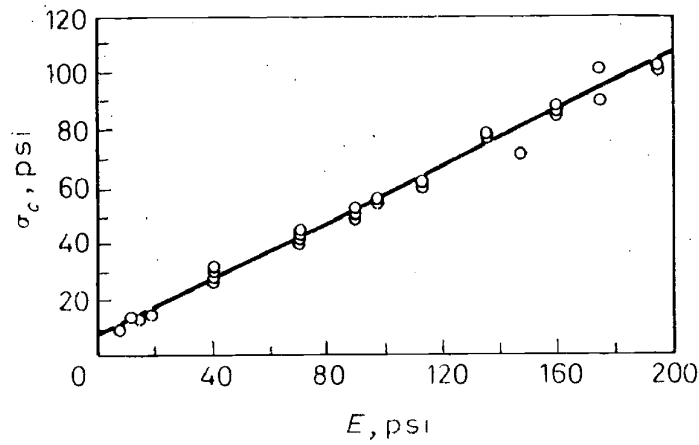

Figure 3. Relation of Young's modulus $E$ for elastomers to the uniaxial tensile stress at the instant of internal rupture in specimens containing a rigid sphere (from Oberth and Bruenner ${ }^{26}$ ). 
cavity in an incompressible medium under hydrostatic tension was considered $^{25}$ in terms of an energy criterion.

Information on rupture in triaxial tension has a direct bearing on rupture processes in filled, and possibly in homogeneous, polymers. As part of a study of filled elastomers, Oberth and Breunner ${ }^{26}$ carried out uniaxial tensile tests on long specimens of polyurethane elastomers in which a large steel sphere was embedded. Owing to the high rigidity of the sphere, the lateral contraction of the specimen near a sphere was partially restrained; thus, regions of triaxial tension developed in the rubber above and below the poles of the sphere. During a test, cavities were observed to form in the rubber (not at the interface) where the triaxial tension was large. The nominal stress on the specimen at the instant of cavitation was found to increase linearly with Young's modulus of the rubber, as shown in Figure 3, the results being similar to those shown in Figure 2.

During a tensile test on elastomers filled with particles whose diameters are greater than, say, a few microns, vacuoles develop around the filler particles (a process called dewetting, see refs. ${ }^{27}$ and ${ }^{28}$ and references cited therein). Based on the observations of Oberth and Bruenner ${ }^{26}$, a cavity undoubtedly first forms near a particle, the cavity then enlarges until it touches the particle, and finally the rubber is pealed from the particle by lateral forces. During a tensile test on a rubber vulcanizate filled with reinforcing carbon black, the observed volume increase, which is related to the degree of dewetting, is very small ${ }^{29}$. However, because the increase in volume is somewhat larger than that for unfilled vulcanizates, it is quite probably that cavities form but that the local energetic conditions (cavity size and elastic energy density) are not sufficient to cause cavity disruption and vacuole formation.

\section{STRENGTH OF ELASTOMERS}

An elastomer is a unique material in that the polymeric chains are highly mobile, yet a deformed specimen regains its original dimensions when the applied stress is removed. Such behaviour results because viscous flow is prevented by chemical crosslinks and permanently entangled chains; the retractive force is provided by the increased conformational free energy (predominantly entropic) of deformed polymeric chains. Although toughness necessitates chain mobility, the mobility in elastomers under most conditions of practical interest is much too large to impart toughness and strength, unless special reinforcing mechanisms become operative. The reason for this characteristic and the source of strength, toughness, and extensibility in different types of elastomers will now be considered. Toughness will not be discussed explicitly because those factors that give a substantial increase in strength also increase the toughness; exceptions to this general rule are probably of limited significance.

In the following discussion, $\sigma_{b}$ and $\lambda_{b} \sigma_{b}$ designate the tensile strengths based on the cross-sectional area of an undeformed and a deformed specimen, respectively; $\lambda_{b}$ and $\lambda_{b}-1$ are, respectively, the extension ratio and the slongation (or strain) at rupture.

We first consider amorphous (noncrystallizable) unfilled elastomers, 
exemplified by a styrene-butadiene rubber (SBR) vulcanizate. At an elevated temperature and a low extension rate, the tensile strength of such an elasto$\mathrm{mer}^{30,31}$ is ordinarily no greater than $100 \mathrm{psi}\left(\mathrm{ca} .7 .0 \mathrm{~kg} / \mathrm{cm}^{2}\right)$ and the elongation-at-break is commonly less than 100 per cent; under such conditions, however, the extensibility is quite dependent on crosslink density, in contrast to the tensile streng th ${ }^{32}$. With a progressive decrease in temperature, the ultimate elongation, $\lambda_{b}-1$, increases 5 - to 10 -fold and then decreases rapidly as the glassy state is approached; concomitantly, the tensile strength increases about 100 -fold. An illustration is provided by Figure 4 in which $\log 273 \sigma_{b} / T$ is plotted against $\log \left(\lambda_{b}-1\right)$ where $T$ is the test temperature in

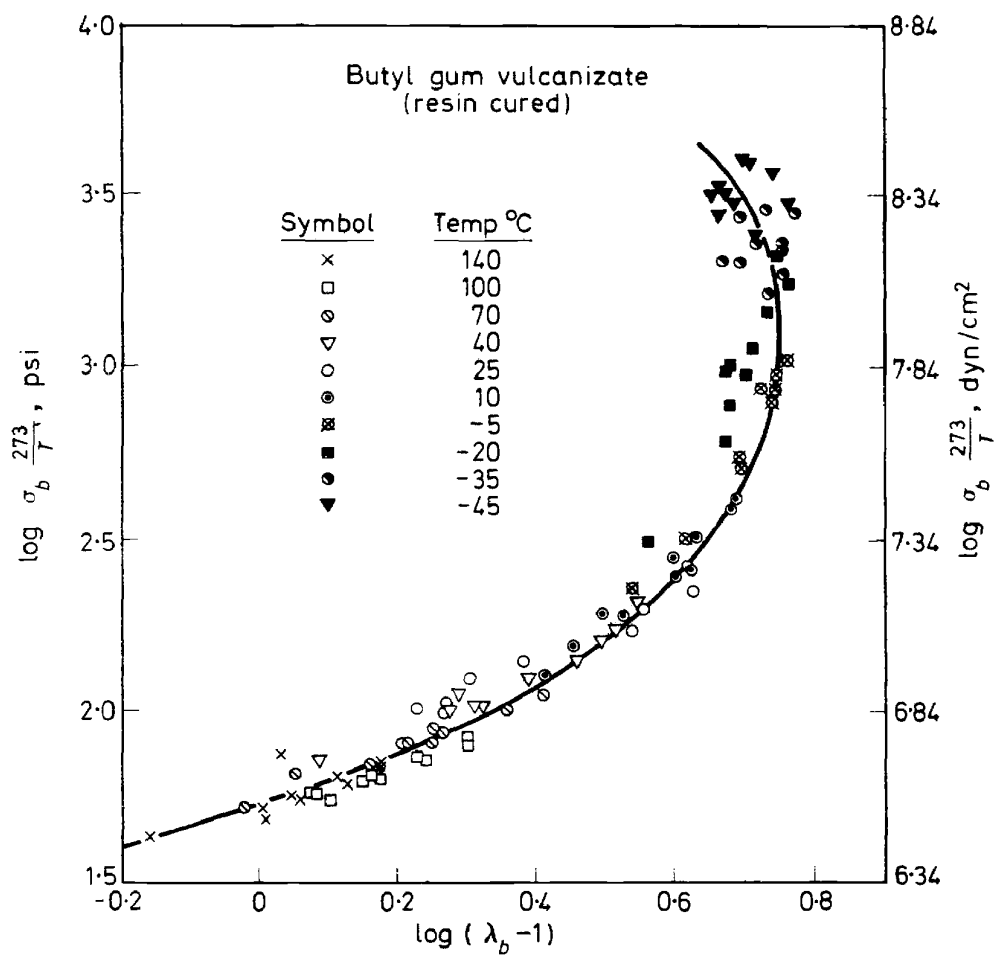

Figure 4. Failure envelope for an unfilled butyl rubber vulcanizate. Temperature-reduced tensile strength, $273 \sigma_{b} / T$, is plotted against the ultimate strain, $\lambda_{b}-1$, on doubly logarithmic coordinates.

${ }^{\circ} \mathrm{K}$. The data were obtained ${ }^{33}$ on an unfilled butyl vulcanizate at various extension rates in the temperature range -45 to $140^{\circ}$. (Under stress, this particular butyl vulcanizate does not crystallize to a significant degree.)

Data measured at different extension rates and temperatures can be superposed ${ }^{30-33}$ to yield master curves that are functions of the temperaturereduced extension rate, $\lambda a_{T}$, or the temperature-reduced time-to-break, $t_{b} / a_{T}$, where $a_{T}$ is the well-known shift factor ${ }^{34}$, which essentially equals the 
mobility of a polymeric segment at an arbitary reference temperature divided by its mobility at temperature $T$. Thus it follows that the temperature dependence of the ultimate properties is a reflection of the change in molecular mobility; likewise, the rate dependence of the ultimate properties results from the local viscous resistance that opposes the stress-biased migration of chains. In other words, the time (rate) and temperature dependence of the ultimate properties should be relatable to the viscoelastic properties of the material.

A semiquantitative relation has been provided by the Bueche-Halpin theory ${ }^{35}$ of rupture. This theory is based on the assumption that the rate of crack growth, during the slow growth stage, is controlled by the viscoelastic response of material near the crack tip. An underlying assumption, introduced for simplicity, is that the crack in a specimen under a constant load grows at some constant (average) rate until a criterion for high speed growth is satisfied. Such considerations lead to :

$$
\sigma_{b}\left(t_{b} / a_{T}\right)=\mathrm{K} / D\left(t_{b} / \mathrm{q} a_{T}\right)
$$

where $\sigma_{b}\left(t / a_{T}\right)$ is the rupture stress when the time-to-break is $t_{b} / a_{T}, \mathrm{~K}$ is a constant (or slowly varying parameter) which is close to unity, and $D\left(t / q a_{T}\right)$ is the creep compliance evaluated at time $t_{b} / \mathrm{q} a_{T}$. The constant $\mathrm{q}$, which is several orders of magnitude greater than unity, is introduced to relate the time-scale for macroscopic creep to that for the creep processes at the crack tip. The assumption that the rate of crack growth in a specimen under a constant load is controlled by viscoelastic (energy dissipative) processes until an instability (high-rate propagation) develops is consistent with observations on the growth of cuts in sheets of rubber $8,14,15$ and with the rate and temperature dependence of the tearing energy ${ }^{36}$.

Owing to the low strength of noncrystallizable elastomers under many test conditions, they are commonly filled with a reinforcing carbon black to obtain increased strength and toughness. The carbon black broadens the relaxation spectrum (or equivalently, the creep compliance curve) and also shifts the spectrum toward longer times or higher temperatures. That is, the carbon black gives a broadening and an upward shift in the time-scale and temperature range within which substantial energy is dissipated in a stressed specimen. In terms of the Bueche-Halpin theory, this modification in viscoelastic properties accounts for the reinforcement effected by carbon black ${ }^{37}$.

In addition to modifying viscoelastic properties, carbon black provides other, or related, mechanisms for reinforcement ${ }^{38,39}$. Under the triaxial stress near filler particles, multiple cavities may form, especially when the stress field in front of a growing crack overlaps the regions of stress concentration near filler particles. This internal breakdown ahead of a crack may: (1) temporarily stabilize a crack owing to the reduction in stored elastic energy and to the possible blunting of the crack tip; and (2) cause the original crack to branch upon further growth, thus dissipating additional energy. The controlling effect of energy dissipation on the strength of unfilled and filled elastomers is clearly shown by the finding that, over a wide temperature range, the energy required to break a specimen is a simple function of a measure of the hysteresis ${ }^{40}$. 
When a natural rubber vulcanizate is stretched, crystallization usually begins at about 300 per cent elongation. Under the very large deformations near the tip of a crack, or in a weak region, crystallization will presumably occur preferentially. The crystallization not only reduced the density of elastic energy but more importantly provides an interface that impedes crack growth. Quite probably, the development of incipient or actual cracks is prevented by local crystallization even before a substantial degree of crystallinity develops in the specimen. In effect, crystallization is a mechanism for self-reinforcement. It probably occurs first in the weakest regions, or near small cracks; subsequently, as other cracks tend to form, their growth is blocked by crystallization. When the elastic energy, or stress, throughout the semicrystalline material finally becomes sufficiently intense, a crack must penetrate crystalline domains. Such a disruptive process, which dissipates considerable energy in plastic deformation and ductile rupture of domains, undoubtedly must occur before a crack and the elastic stored energy become sufficiently large to satisfy a criterion, like equation 2 , for self-sustained crack growth.

At elevated temperatures, crystallization apparently does not occur to a significant degree in a natural rubber vulcanizate. Under such conditions, the strength and extensibility depend strongly on extension rate and temperature ${ }^{32,33}$, as is characteristic of noncrystallizable elastomers. Also, from tests at ambient temperature, it has been found ${ }^{41,42}$ that, with a progressive increase in extension rate, the tensile strength passes through a maximum and then begins to increase again. The decrease in strength results because crystallization does not have time to occur during the test period ; the subsequent increase is attributed to the increased viscous dissipation of energy at the very high extension rates.

Figure 5 shows the temperature dependence of the rupture stress $\lambda_{b} \sigma_{b}$ (based on the cross-sectional area of the deformed specimen) and the ultimate elongation $\lambda_{b}-1$ for unfilled vulcanizates of SBR and butyl rubber at an extension rate of $1 \mathrm{~min}^{-1}$. The abrupt increase in $\lambda_{b} \sigma_{b}$ and $\lambda_{b}-1$ for the butyl vulcanizate at slightly below $40^{\circ}$ is attributed ${ }^{32,33}$ to the onset of crystallization. That is, at the lower temperatures, some crystallization undoubtedly occurs, blocking crack growth; at higher temperatures, the strength and ultimate elongation are low because little or no crystallization occurs.

Elastomeric styrene-butadiene-styrene triblock polymers, exemplified by Kraton 101 and Thermolastic 226 manufactured by the Shell Chemical Company, are two-phase materials consisting of glassy polystyrene domains (islands) embedded in an elastomeric polybutadiene matrix. For Kraton $101, \lambda_{b} \sigma_{b}$ and $\lambda_{b}-1$ are exceptionally high and nearly temperature independent below about $40^{\circ}$, as shown in Figure 5. The high strength arises ${ }^{43}$ from the large amount of energy dissipated in the vicinity of slowly growing cracks; the dissipation results from the plastic deformation and ductile rupture of the polystyrene domains. Such a process presumably must occur before a crack achieves a critical size required for initiation of high speed growth. The properties of a plasticized triblock polymer (Thermolastic 226) are also shown in Figure 5. The substantially reduced tensile strength above $0^{\circ}$ is attributed primarily to the plasticizer which presumably softens the 

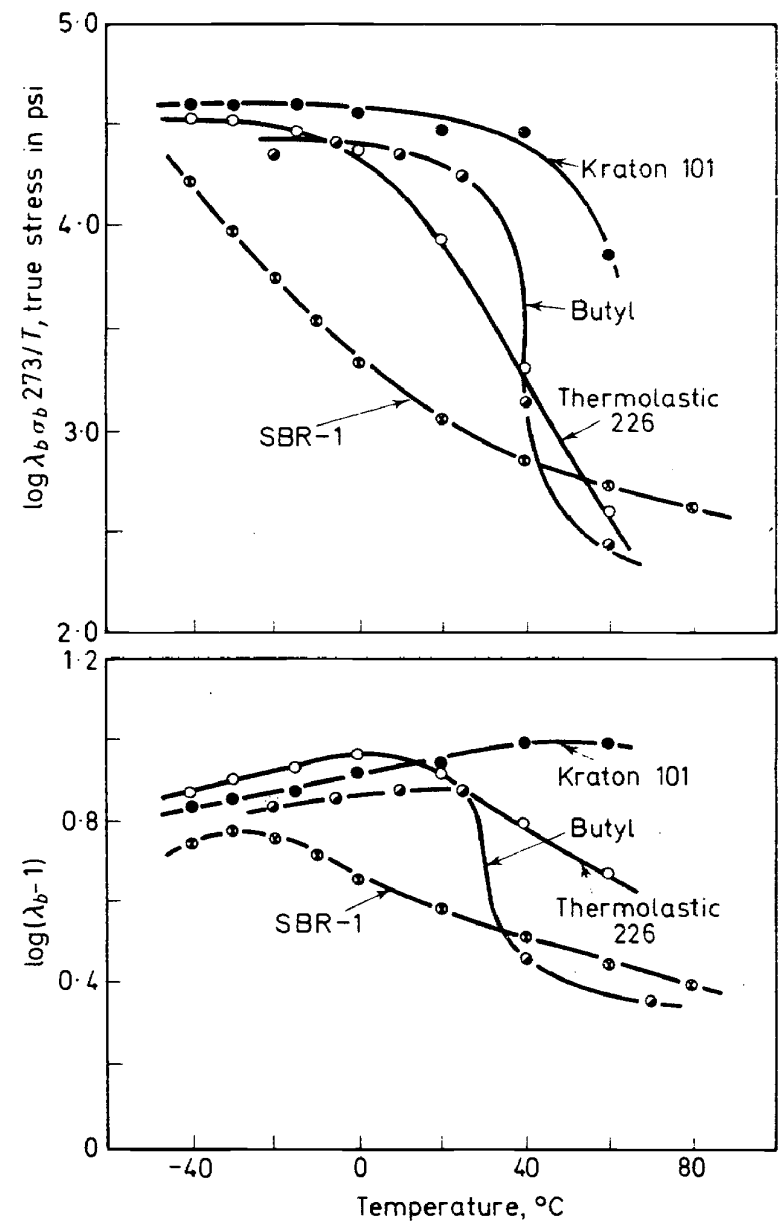

Figure 5. Temperature dependence of the ultimate tensile properties to two elastomeric triblock polymers (Kraton 101 and Thermolastic 226) and unfilled vulcanizates of styrene-butadiene and butyl rubbers. The tensile strength $273 \lambda_{b} \sigma_{b} / T$ is based on the cross-sectional area of the deformed specimen. Data are at an extension rate of $1 \mathrm{~min}^{-1}$

domains or the diffuse interfacial regions between domains and the matrix.

Recently, the rupture of an unfilled SBR vulcanizate in equal biaxial tension (EBT) has been studied by inflating a membrane into a bubble ${ }^{44}$ Quite surprisingly, $\lambda_{b}$ was found to be nearly independent of extension rate and temperature in the range -43 to $50^{\circ}$. Above $-15^{\circ}, \lambda_{b}$ was substantially greater than in simple tension (ST). Furthermore, over nearly the entire temperature range, the tensile strength was found to be greater, and also considerably less dependent on extension rate and temperature, than in ST. Actually, however, the data reported are considered to represent behaviour in EBT only approximately; rupture did not occur near the pole of the bubble, the only region in which the stress state is precisely EBT. 
From one viewpoint, the extensibility in EBT would be expected to be considerably less than in ST, contrary to the experimental results. At a given strain, as already discussed, the homogeneous dilatation (Table 2) and also the elastic stored energy is considerably greater in EBT than in ST. For a neo-Hookean rubber at $\lambda=3$, the stored energy in EBT is about two-fold greater than in ST. Hence, the tendency to cavitate should also be greater. However, cavity formation cannot be equated to macroscopic rupture; unless a cavity is converted into a growing crack, cavitation may stabilize a material by relieving unfavourable regions of stress concentration.

During the tests in EBT, it is quite possible that cavities did in fact form in the region of EBT but they did not transform into cracks because a preferred direction for crack growth did not exist. Normally, a crack propagates perpendicular to the maximum principal stress; in equal biaxial tension, two principal stresses are equal and thus a preferred direction for growth does not exist. For this reason, rupture began in a region of unequal biaxial tension. From an examination of the rupture pattern, it appears ${ }^{45}$ likely that rupture resulted from the formation of a relatively large blister on the side of the bubble (an instability that is not surprising) and the subsequent propagation of a crack around the base of the blister.

The study of rupture in EBT illustrates that the rate controlling step in the rupture process can depend markedly on the stress state. In simple tension, the controlling step is the slow growth of cracks. When rupture is induced to occur under a stress state that is precisely equal biaxial tension (not achieved in the above-mentioned study), it seems quite probable that the slow growth step is largely, if not completely, bypassed.

In summary, the discussion on strength of elastomers clearly indicates that only those materials which contain a dispersed phase, or under stress develop a second phase, exhibit high strength over broad ranges of temperature and time. Except at relatively low temperatures, amorphous elastomers are weak unless a reinforcing filler is present; crystallizable elastomers are strong because of the self-reinforcement that results from the stress-induced formation of crystalline domains; and the high strength of elastomeric triblock, and certain segmented (alternating block) elastomers results from the plastic domains which, like crystalline regions, function to impede the growth of cracks to a critical size in the relatively soft, liquid-like matrix.

\section{PLASTICS AND GLASSY POLYMERS}

A highly desired characteristic of plastics and glassy polymers is toughness, commonly specified in terms of impact strength. Such a characteristic results from energy dissipation during the plastic deformation that accompanies either of two types of yield processes ${ }^{46,47}$ : yielding in shear, and yielding under normal stresses. The latter is a dilatational process commonly referred to as craze formation. As already mentioned, crazing involves cavitation followed by a plastic deformation of the cavities to give an oriented foam-like structure in limited regions of the specimens. The diameter of the pores or channels in a craze is very small, being in the order of $200 \AA$; the void content is typically in the range $40-60$ per cent ${ }^{48}$.

A detailed study $y^{46,47}$ has been made of poly (methyl methacrylate) to 
determine the stress states which bring about crazing and shear yielding. Under all states of biaxial tension, it was concluded that crazing occurs at lower stresses than those required to give shear yielding. However, when the principal stresses are such that the first stress invariant $\left(I_{1}=\sigma_{1}+\sigma_{2}+\sigma_{3}\right)$ is less than some value, shear yielding tends to occur preferentially. Under pure shear conditions, at which the dilatation is zero, i.e. $I_{1}=0$, shear yielding occurs. For $I_{1}>0$, the tensile stress required to give shear yielding is a decreasing function of $I_{1}$; this behaviour results from the increase in the free volume, and thus in the mobility, effected by the dilatative stress state. Based on experimental data, the yield stress in simple compressive was predicted ${ }^{47}$ to be about 30 per cent greater than in simple tension, in agreement with results from an earlier study of dilatation and yielding in glassy polymers ${ }^{49}$.

Under many test conditions, the states of stress and strain throughout a specimen are not known. Commonly, the stress is nonhomogeneous owing to heterogeneities within a specimen and also to end-effects which subject the test section to elastic constrains. When elastic constraints exist, test data ordinarily depend on specimen geometry. Similarily, the stress state in the vicinity of a crack, or plastically yielded material, often depends on specimen dimensions.

To illustrate the dependence of test results on specimen dimensions, some experiments made on a polycarbonate plastic by Morecroft ${ }^{50}$ will be briefly mentioned. In his study, Izod impact tests were conducted on notched specimens differing in thickness. When the thickness was less than about 0.2 inch, the impact strength (toughness) was nearly $10^{8} \mathrm{ergs} / \mathrm{cm}^{2}$. On the other hand, when the thickness was greater than about $0 \cdot 2$ inch, the toughness was lower by a factor of 5 to 10 , depending on the exact thickness. In a thin specimen, a zone of plastically deformed material develops ahead of the crack and absorbs considerably energy as the crack propagates. However, when the thickness of a specimen is greater than some critical value, the lateral contraction that accompanies a plastic deformation is largely prevented by the sizeable volume of elastic material that surrounds the crack front. As plastic yielding ahead of the crack is thus largely impeded, the crack propagates brittly with relatively little energy being dissipated.

The critical thickness at which the toughness (impact strength) drops precipitously was studied ${ }^{50}$ as a function of the rate at which the specimen was deformed. Figure 6 (reproduced from ref. ${ }^{51}$ ) shows that the critical thickness increases substantially as the imposed velocity is decreased from $10^{3}$ to $10^{-4} \mathrm{ft}$./ $/ \mathrm{sec}$. Under the conditions represented by the region to the left of the line in Figure 6, the material is tough, whereas to the right of the line, the material is relatively brittle. These results also indicate that yielding is a time-dependent process.

The effect of elastic constraints is also illustrated by results from uniaxial tensile tests made $^{50}$ on cylindrical specimens having length-to-diameter ratios of about 4 and 6 . The specimen having a length-to-diameter ratio of 6 underwent yielding and broke at a relatively high elongation. In contrast, the other specimen broke at a relatively low elongation without yielding. This behaviour results from elastic constraints arising because of the small length-to-diameter ratio of the specimen.

Rubber particles are commonly dispersed in brittle plastics to increase 
impact strength, or equivalently, the fracture energy ${ }^{52-58}$ To obtain improved toughness, it is necessary that the volume fraction as well as the diameter of the particles be in some suitable range and that the particles be firmly bound to the plastic matrix ${ }^{52,56,57}$

When the temperature of a toughened specimen is progressively decreased

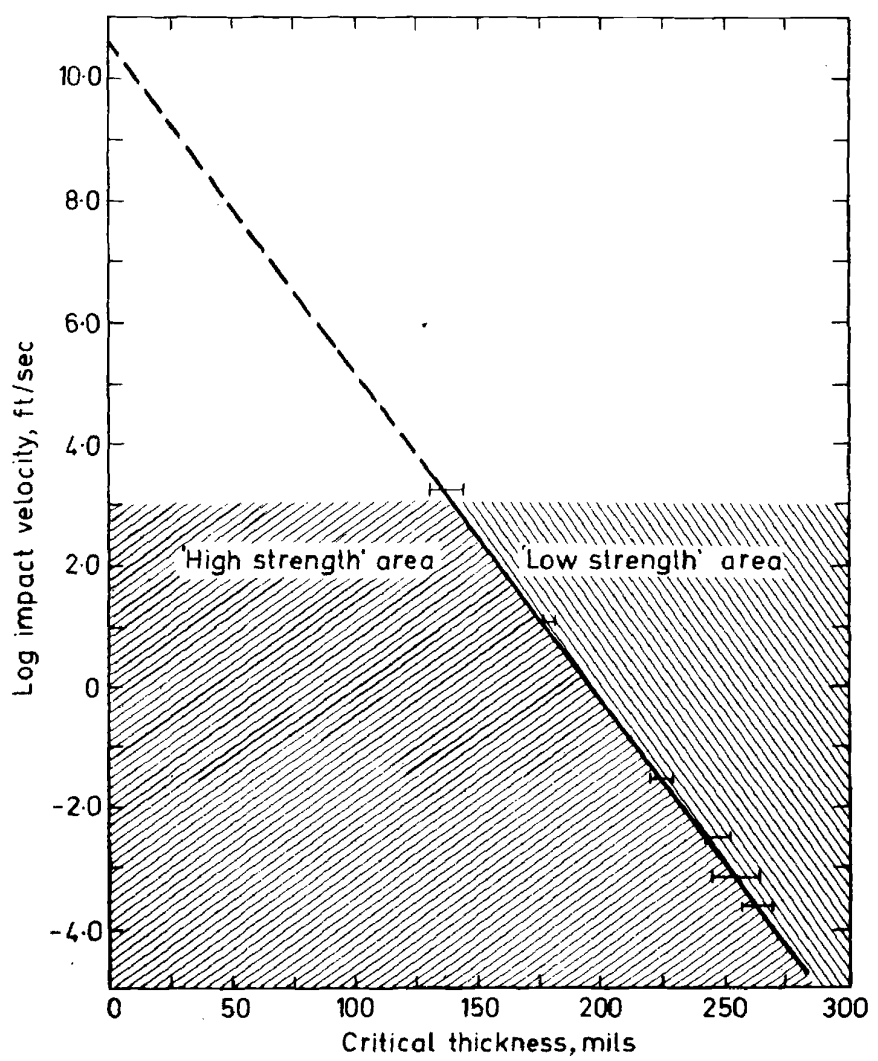

Figure 6. Dependence of thickness at which the strength of a notched specimen decreases markedly on the logarithm of the impact velocity. Data on a polycarbonate resin ${ }^{50,51}$

from the molding temperature, dilatation under triaxial tension develops in the rubber and certain regions of the matrix because the thermal expansion coefficient of rubber is about threefold greater than that of a plastic. In addition, when the specimen is subjected to a uniaxial tensile load, a relatively large dilatation is produced in the matrix near the equatorial plane of each particle; the triaxial tension in the rubber is also increased. This behaviour arises because rubber tends to deform at constant volume whereas the volume of a plastic increases during a tensile deformation. Hence, if the rubber particles are bonded to the matrix, triaxial tension develops in both the 
rubber and the plastic matrix. The induced dilatation promotes the formation of crazes, or possibly microcracks, in the vicinity of the particles, a process that dissipates considerable stored elastic energy. If the concentration and size of the rubber particles are in a certain range, the growth of a large craze or crack is prevented by the intersection of a growing crack with a particle in the vicinity of the initiation site. Specifically, as a crack forms and grows, a zone of crazed material continues to form in front of the crack. If the width of the zone is large compared to the size of the rubber particles, a growing crack will not be blocked by the particles. However, if the rubber particles are relatively large, the crack will be arrested; other cracks will then grow until blocked by particles. Eventually, however, some crack will become sufficiently large to satisfy a criterion for self-sustained high-speed growth. In a study ${ }^{57}$ of epoxy resins in which a low molecular weight rubber is dispersed, it was found that no toughening occurs when the dispersed rubber particles are less than about $1000 \AA$ in diameter.

\section{CONCLUSIONS}

Rupture processes involve an initiation phase, the slow-growth of cracks, and high-speed crack propagation. Initiation consists of cavitation or the activation of pre-existent cavities or cracks. The mode and rate of crack development, prior to catastrophic growth, are affected by the stress state and the dissipation rate of elastic energy stored near a crack, as well as the physical characteristics of the material. The stress near a crack depends not only on its size and the surface tractions applied to the specimen but also on the number, size and distribution of heterogeneities, included cavities and other cracks. Under certain conditions, the growth rate of a particular crack may be decreased, or even arrested, by material heterogeneities or the nearby development of cavities or cracks. High-speed crack growth apparently occurs when the product of the elastic energy density and the crack size exceeds a critical value that depends on material characteristics and experimental conditions. These qualitative remarks about microscopic breakdown indicate that strength, and especially toughness, are controlled by dissipative and disruptive processes that operate to relieve unfavourable stress states, thus reducing the elastic energy density, in localized regions of a specimen. Examples of such processes are cavitation and craze formation, viscoelastic and plastic deformations near growing cracks, and stress-induced crystallization.

From a consideration of rupture processes, it can be concluded that all polymeric materials which exhibit high strength and toughness over extended ranges of time and temperature have two phases or develop a second phase under stress. To obtain optimum properties, the structural characteristics of the material must be such that many small cavities, crazes, or cracks form but that mechanisms then come into play to impede their continued growth. If only a few cracks develop, a particular crack can more readily attain a critical size, thus allowing rupture to occur with the expenditure of considerably less energy, than if numerous crazes or cracks form throughout a specimen. 


\section{ACKNOWLEDGEMENT}

The author wishes to acknowledge the use of an unpublished manuscript kindly furnished by the late A. S. Morecroft of the Mobay Chemical Company.

\section{References}

1 V. R. Regel, T. M. Muinov, and O. F. Pozdnyakov. Physical Basis of Yield and Fracture: Conference Proceedings (Oxford, September 1966), Institute of Physics and Physical Society, Conference Series No. 1, London, p. 194.

2 E. H. Andrews and P. E. Reed. J. Polymer Sci. B, 5, 317 (1967).

${ }^{3}$ S. N. Zhurkov and E. E. Tomashevsky. Physical Basis of Yield and Fracture; Conference Proceedings (Oxford, September 1966), Institute of Physics and Physical Society, Conference Series No. 1, London, p. 200.

4 D. Campbell and A. Peterlin. J. Polymer Sci. B, 6, 481 (1968).

5 K. L. DeVries, E. R. Simonson, and M. L. Williams. Am. Chem. Soc. Polymer Preprints 10, $1190(1969)$.

6 J. S. Ham, unpublished results.

7 V. R. Regel. Soviet Physics (Tech. Phys.) 1, 353 (1956).

8 W. G. Knauss. Trans. Soc. Rheology 13, 291 (1969).

9 C. J. Phillips. American Scientist 53, 20 (1965).

$10 \mathrm{G}$. Kraus, unpublished results.

11 J. Cook and J. E. Gordon. Proc. Roy. Soc. A, 282; 508 (1964).

12 H. Schardin. Fracture, Ch. 16, B. L. Averbach et. al., Eds., Wiley, N.Y. 1959.

13 P. Mason. J. Appl. Phys. 29, 1146 (1958).

14 W. Knauss. The Time-Dependent Fracture of Viscoelastic Materials. Proc. I.Intern. Congress Fracture Sendai, Japan, 1965.

15 H. W. Greensmith. J. Appl. Polymer Sci. 8, 1113 (1964).

16 I. Wolock and S. B. Newman Fracture Processes in Polymeric Solids, Ch. IIC, B. Rosen, Ed., Interscience (Wiley), N.Y. 1964.

17 B. Rosen. Fracture Processes in Polymeric Solids, Ch. IIIA, B. Rosen, Ed., Interscience (Wiley), N.Y. 1964.

18 B. Maxwell and L. F. Rahm. Ind. Eng. Chem. 41, 1988 (1949).

19 K. B. McAfee, Jr., J. Chem. Phys. 28, 218, 226 (1958).

20 B. Rosen. J. Polymer Sci. 47, 19 (1960).

21 S. N. Zhurkov, V. A. Marikhan, and A. I. Slutsker. Soviet Phys.-Solid State 1, 1060 (1960).

22 R. N. Haward. Trans. Faraday Soc. 38, 394 (1942).

23 A. N. Gent and P. B. Lindley. Proc. Roy. Soc. A, 249, 195 (1958).

${ }^{24}$ G. H. Lindsey. J. Appl. Phys. 38, 4843 (1967).

25 M. L. Williams and R. A. Schapery. Fracture Mechanics 1, 64 (1965).

26 A. E. Oberth and R. S. Bruenner. Trans. Soc. Rheology 9 (Part 2), 165 (1965).

27 T. L. Smith. Trans Soc. Rheology 3, 113 (1959).

28 R. J. Farris. Trans. Soc. Rheology 12, 315 (1968).

29 R. Shuttleworth. European Polymer J. 12, 315 (1968).

30 T. L. Smith. J. Polymer Sci. 32, 99 (1958).

31 T. L. Smith. J. Polymer Sci. A1, 3597 (1963).

32 T. L. Smith. Strength and Extensibility of Elastomers, Rheology, Vol. 5, Ch. 4. F. R. Eirich, Ed. Academic Press, New York, 1969.

33 T. L. Smith. J. Appl. Phys. 35, 27 (1964).

34 J. D. Ferry. Viscoelastic Properties of Polymers, Wiley, N.Y., 1961.

35 J. C. Halpin. J. Appl. Phys. 35, 3133 (1964); Rubber Chem. Technol. (Review Issue) 38, 1007 (1965).

36 H. W. Greensmith, L. Mullins, and A. G. Thomas. The Chemistry and Physics of RubberLike Substances, Ch. 10, L. Bateman, Ed., Wiley, N.Y., 1963.

37 J. C. Halpin and F. Bueche. J. Appl. Phys. 35, 3142 (1964).

38 E. H. Andrews and A. Walsh. J. Polymer Sci. 33, 39 (1958); Proc. Phys. Soc. (London) 72, 42 (1958).

39 E. H. Andrews. Rubber Chem. Technol. 34, 325 (1963). 


\section{RUPTURE PROCESSES IN POLYMERS}

40 K. A. Grosch, J. A. C. Harwood, and A. R. Payne. Physical Basis of Yield and Fracture: Conference Proceedings (Oxford, September 1966), Institute of Physics and Physical Society, Conference Series No. 1, London, p. 144.

41 C. N. Zhurkov, T. P. Sanfirova, and E. E. Tamashevskii. Rubber Chem. Technol. 35, 813 (1962).

42 G. Fromandi, R. Ecker, and W. Heidemann. Kautschuk und Gummi 13(2), WT 25 (1960).

43 T. L. Smith and R. A. Dickie, Block Copolymers (J. Polymer Sci. C, 26) J. Moacinin, G. Holden, and N. W. Tschoeg1, Eds., Interscience, New York, p. 163 (1969).

44 R. A. Dickie and T. L. Smith. J. Polymer Sci. A-2, 7, 687 (1969).

45 F. R. Eirich, private communication.

${ }^{46}$ S. S. Sternstein, L. Ongchin, and A. Silverman. Applied Polymer Symposia: Polymer Modification of Rubbers and Plastics, Vol. 7, Interscience, New York, p. 175 (1968).

47 S. S. Sternstein and L. Ongchin. Am. Chem. Soc. Polymer Preprints 10, 1117 (1968).

48 R. P. Kambour. Applied Polymer Symposia: Polymer Modification of Rubbers and Plastics, Vol. 7, Interscience, New York, p. 215 (1968).

49 W. Whitney and R. D. Andrews. International Symposium on Macromolecular Chemistry (J. Polymer Sci. C, 16) O. Wichterle and B. Sedlacek, Eds., Interscience, New York, p. 2981 (1967).

50 A. S. Morecroft, unpublished results.

51 F. R. Eirich. Applied Polymer Symposia; High Speed Testing: The Rheology of Solids, Vol. 5, R. D. Andrews, Jr. and F. R. Eirich, Eds., Interscience, New York, p. 271 (1967).

52 R. N. Haward and J. Mann. Proc. Roy. Soc. A 282, 120 (1964).

53 C. B. Bucknall and R. R. Smith. Polymer 6, 437 (1965).

54 S. Newman and S. Strella. J. Applied Polymer Sci. 9, 2297 (1965).

55 S. Strella. Applied Polymer Symposia: Polymer Modification of Rubbers and Plastics, Vol, 7, Interscience, New York, p. 165 (1968).

56 J. A. Schmitt. J. Applied Polymer Sci. 12, 533 (1968).

${ }^{57}$ F. J. McGarry and A. M. Miller. Am. Chem. Soc. Preprints of Division of Organic Coatings and Plastic Chemistry 28, 512 (1968).

${ }^{58}$ F. J. McGarry and J. N. Sultan. Am: Chem. Soc. Preprints of Division of Organic Coatings and Plastic Chemistry 28, 526 (1968). 